\title{
Example of Steady-State Electric-Current Modeling of a Complicated Cellular Topology with Boundary Element Fast Multipole Method
}

\author{
Vishwanath Iyer, William A. Wartman, Aapo Nummenmaa, \\ and Sergey N. Makarov
}

\section{Introduction}

The recently developed quasistatic formulation of the boundary element fast multipole method or BEM-FMM [1, 2] is based on the integral equation written in terms of induced charge density at the interfaces, which is naturally coupled with the general-purpose fast multipole method [3, 4]. It could potentially be applied to perform quasistatic electromagnetic modeling of complicated 3D surface topologies on the cellular level. Modeling such topologies with the finite element method or FEM is hardly possible in practice, in particular due to the complexity of creation of the required volumetric tetrahedral mesh and the required high mesh resolution near multiple fine model joints.

\footnotetext{
V. Iyer $(\bowtie)$

The MathWorks, Inc., Natick, MA, USA

e-mail: vishwanath.iyer@mathworks.com

W. A. Wartman

Electrical and Computer Engineering Department, Worcester Polytechnic Institute,

Worcester, MA, USA
}
A. Nummenmaa
Athinoula A. Martinos Center for Biomedical Imaging, Massachusetts General Hospital, Charlestown, MA, USA
S. N. Makarov
Electrical and Computer Engineering Department, Worcester Polytechnic Institute,
Worcester, MA, USA
Athinoula A. Martinos Center for Biomedical Imaging, Massachusetts General Hospital, Charlestown, MA, USA




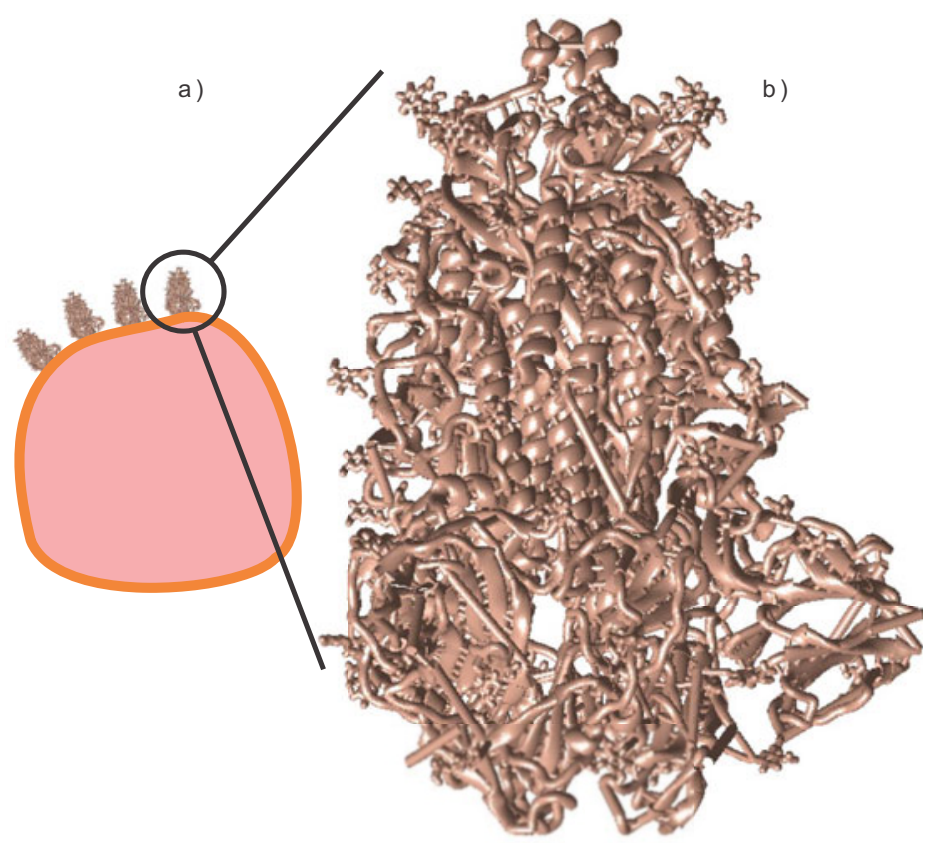

Fig. 1 CAD-based SARS-CoV-2 spike glycoprotein structure [5]. No differentiation between different protein types is made

In this study, we report the performance of the BEM-FMM algorithm for classic (quasi) steady-state electric current modeling around a CAD-based SARS-CoV-2 spike glycoprotein structure [5]. This structure is part of the mechanism by which the coronavirus attaches to a target object, see Fig. 1a. The target object itself is not included into the present study; only the conceptual possibility of quasistatic modeling and the method's numerical performance are reported and discussed.

\section{Materials and Methods}

Figure 1b shows the protein CAD model [5]. While the overall SARS-CoV-2 virus particle size is reported to be in the range of 80-200 $\mathrm{nm}[6,7]$, the spike glycoprotein structure shown in Fig.1a is much smaller, possibly in the range of 10-20 nm [8]. The electrical conductivity of protein may vary [9]; we assume that the macroscopic conductivity of protein approaches zero while the conductivity of the ambient body fluid is $0.1 \mathrm{~S} / \mathrm{m}$. A primary electric field with the amplitude of $1 \mathrm{~V} / \mathrm{m}$ is applied along the x-axis, as shown in Figs. 2 and 3, respectively. 


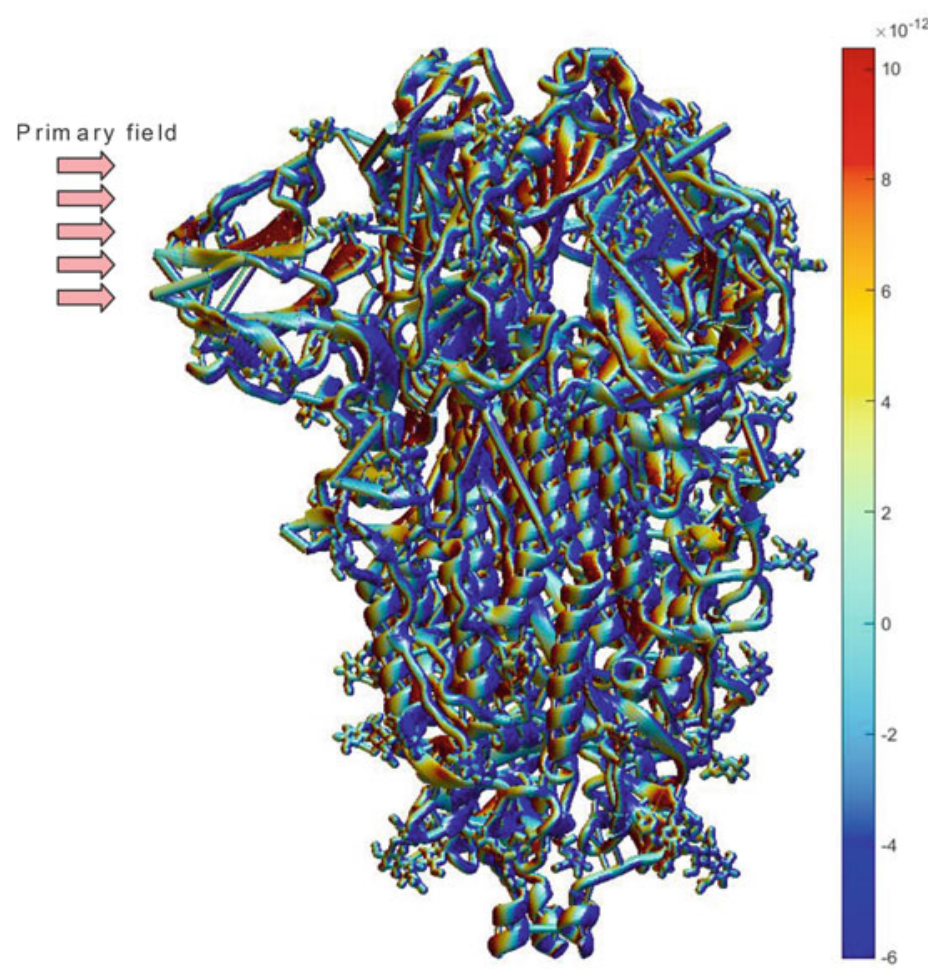

Fig. 2 Primary incident electric field and surface charge density distribution in $\mathrm{C} / \mathrm{m}^{2}$ on the surface of the CAD object

The entire surface CAD model has $2.34 \mathrm{M}$ facets. Nine facets were found to have coincident face centers, although their vertices were somewhat different. These duplicated facets were removed from the mesh prior to simulation.

\section{Results}

The numerical solution with 100 GMRES iterations executes in approximately 10 min using a general-purpose Intel Xeon E5-2698 v4 CPU (2.10 GHz) Windows server, 256 GB RAM, which runs MATLAB 2019b. The final relative residual of the iterative solution is $4 \times 10^{-7}$; the charge conservation law is satisfied to within a $10^{-5}$ relative error. 


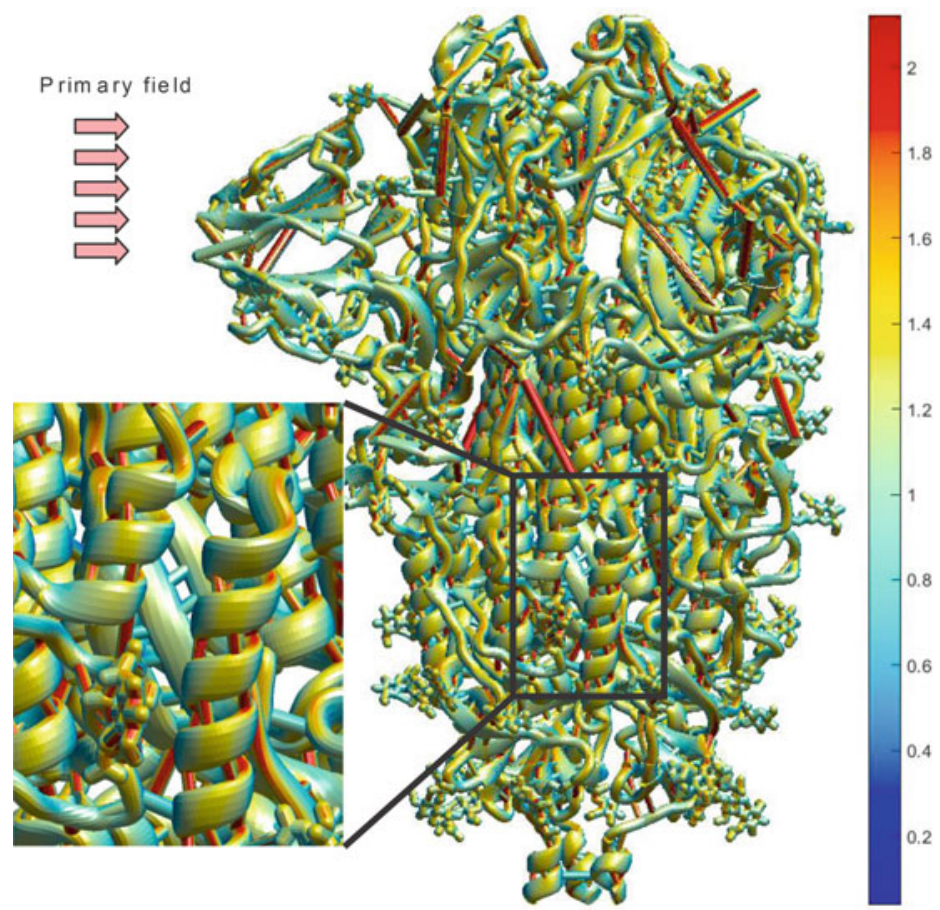

Fig. 3 Total electric field distribution just outside the surface of the CAD object in V/m. Note the inset which shows the zoomed-in field distribution

Figure 2 shows the direction of the primary electric field and the resulting distribution of the surface charge density in $\mathrm{C} / \mathrm{m}^{2}$ on the surface of the CAD object subject to the applied electric field. There seems to be no dedicated domain where the absolute charge concentration would be the largest.

Similarly, Fig. 3 demonstrates the total electric field distribution just outside the surface of the CAD object in $\mathrm{V} / \mathrm{m}$. A potentially interesting observation is that the total electric field becomes quite large at certain vertical segments of the protein structure (an inset in Fig. 3), which remain largely perpendicular to the primary field. Regarding the surface fields, the simulations reveal that the total field just outside the surface might exceed the primary field by a factor of up to 80 . Further modeling is necessary to justify this result.

The normal field just outside the surface approaches zero (this fact also follows from the current continuity condition) so that the tangential field nearly coincides with the total field. 
We further studied the same problem but at different orientations of the primary electric field. Similar convergence results have been observed, but the field distributions are quite different.

\section{Discussion and Conclusion}

The BEM-FMM approach allows us to model the present rather complicated topology in approximately 10 min using a common Windows server and with a high degree of convergence accuracy. It is straightforward to assign different properties to different constituents of the microscopic structure. It is also possible to clone the structure multiple times and include the effect of a nearby cell object.

Indeed, the performed modeling task remains purely classical and "macroscopic," with no relevant quantum effects included. At the same time, it demonstrates the conceptual possibility of quasistatic modeling via BEM-FMM and demonstrates the method's numerical performance.

\section{References}

1. Makarov, S. N., Noetscher, G. M., Raij, T., \& Nummenmaa, A. (2018). A quasi-static boundary element approach with fast multipole acceleration for high-resolution bioelectromagnetic models. IEEE Transactions on Biomedical Engineering, 65(12), 2675-2683. https://doi.org/10. 1109/TBME.2018.2813261.

2. Htet, A. T., Saturnino, G. B., Burnham, E. H., Noetscher, G., Nummenmaa, A., \& Makarov, S. N. (2019a). Comparative performance of the finite element method and the boundary element fast multipole method for problems mimicking transcranial magnetic stimulation (TMS). Journal of Neural Engineering, 16, 1-13. https://doi.org/10.1088/1741-2552/aafbb9.

3. Greengard, L., \& Rokhlin, V. (1987). A fast algorithm for particle simulations. Journal of Computational Physics, 73(2), 325-348. https://doi.org/10.1016/0021-9991(87)90140-9.

4. Gimbutas, Z., Greengard, L., Magland, J., \& Rachh, M. (2019). Rokhlin V. fmm3D Documentation. Release 0.1.0. 2019. Online: https://github.com/flatironinstitute/FMM3D

5. Hernandez, J. (2020). Structure of the SARS-CoV-2 spike glycoprotein (closed state). Fri, 202003-13 12:38 PM. National Institutes of Health 3D Print Exchange. Online: https://3dprint.nih. gov/discover/3DPX-013123.

6. Chen, N., Zhou, M., Dong, X., Qu, J., Gong, F., Han, Y., Qiu, Y., Wang, J., Liu, Y., Wei, Y., Xia, J., Yu, T., Zhang, X., \& Zhang, L. (2020, Feb 15). Epidemiological and clinical characteristics of 99 cases of 2019 novel coronavirus pneumonia in Wuhan, China: a descriptive study. Lancet, 395(10223), 507-513. Online: https://www.thelancet.com/action/showPdf?pii=S0140-6736\% 2820\%2930211-7. 
7. Kim, J.-M., et al. (2020). Identification of Coronavirus Isolated from a Patient in Korea with COVID-19. Osong public health and research perspectives, 11(1), 3-7. Online: https://www. ncbi.nlm.nih.gov/pmc/articles/PMC7045880/pdf/ophrp-11-3.pdf.

8. Walls, A. C., Tortorici, M. A., Snijder, J., Xiong, X., Bosch, B.-J., Rey, F. A., \& Veesler, D. (2017, Oct). Tectonic conformational changes of a coronavirus spike glycoprotein promote membrane fusion. Proceedings of the National Academy of Sciences, 114(42), 11157-11162. https://doi.org/10.1073/pnas.1708727114. Online: https://www.pnas.org/content/pnas/114/42/ 11157.full.pdf

9. Zhang, X. Y., Shao, J., Jiang, S. X., Wang, B., \& Zheng, Y. (2015 Mar 27). Structure-dependent electrical conductivity of protein: Its differences between alpha-domain and beta-domain structures. Nanotechnology, 26(12), 125702. https://doi.org/10.1088/0957-4484/26/12/125702.

Open Access This chapter is licensed under the terms of the Creative Commons Attribution 4.0 International License (http://creativecommons.org/licenses/by/4.0/), which permits use, sharing, adaptation, distribution and reproduction in any medium or format, as long as you give appropriate credit to the original author(s) and the source, provide a link to the Creative Commons license and indicate if changes were made.

The images or other third party material in this chapter are included in the chapter's Creative Commons license, unless indicated otherwise in a credit line to the material. If material is not included in the chapter's Creative Commons license and your intended use is not permitted by statutory regulation or exceeds the permitted use, you will need to obtain permission directly from the copyright holder. 